\title{
La sombra de la violencia estructural en los jóvenes universitarios
}

\author{
The shadow of structural violence in young students
}

NATALIA IX-CHEL VÁZQUEZ GONZÁLEZ, GUILLERMINA DÍAZ PÉREZ y ARACELI PÉREZ DAMIÁN

UnIVERSIDAd AutónOMa DEL ESTAdo DE MÉxico

Artículo recibido: 5 junio 2014

Solicitud de revisión: 4 noviembre 2014

Artículo aceptado: 13 enero 2015

Resumen

Las crisis económicas están generando desigualdades, producto de la violencia estructural; estas están derivando en las maneras en que los jóvenes construyen y dibujan los sentidos sociales. Los jóvenes deberían ser sujetos que se mueven en toda una temporalidad, sin embargo, sus condiciones materiales de vida no les permiten pensar en un futuro con certezas. Más bien, estas se desdibujan para ellos y hoy más que nunca aparecen sus sueños postergados y aplazados por la propia violencia de la que son objeto. De ahí, nuestro interés en ver cómo los jóvenes egresados de la universidad, están construyendo dichos sentidos.

Palabras clave: jóvenes, violencia estructural, sentidos sociales, futuro.

\begin{abstract}
Economic crises are generating inequalities resulting from structural violence; they are drifting in the ways in which young people build and draw social senses. Young people should be the subjects of movement along temporariness; nevertheless, their material living conditions do not allow them to think in their future with certainty. For our young generations these conditions are blurred, and now more than ever their dreams are deferred and delayed by the violence they are subjected to. Hence, our interest in finding out how young university graduates are building these senses.
\end{abstract}

Keywords: young people, structural violence, social senses, future. 


\section{INTRODUCCIÓN}

Las crisis económicas, políticas, medioambientales y energéticas que vive México están afectando a su población juvenil en las oportunidades que ellos tienen para desarrollar sus capacidades, así por ejemplo, muchos de ellos no tienen acceso a la educación, y aquellos que llegan a cursar una licenciatura no logran insertarse en el mercado profesional. Desde la investigación para la paz, la falta de oportunidades es, lo que se llama, violencia estructural.

Hablar de violencia estructural es hablar de los índices de desigualdad, pero también, es relevante hablar de cómo los sujetos que la viven la significan y resignifican en sus vidas cotidianas, cómo le hacen frente y cómo miran y dibujan su futuro. Para fines del presente trabajo, es importante analizar cómo los jóvenes mexicanos, que han pasado por las aulas universitarias y han concluido una licenciatura, están significando la violencia estructural que se vive en México; bajo el entendido de que comprender las lógicas de producción de sentido que se hace de la violencia estructural, nos remite necesariamente a mirar las prácticas sociales y las significaciones que se hace de su vida misma, de la mirada al futuro, de sus descontentos y de sus reinvenciones frente a las crisis.

Pareciera que los jóvenes mexicanos, egresados de la universidad, no sufren de violencia estructural ni de las desigualdades provocadas por las distintas crisis que configuran la realidad nacional; sin embargo, cuando egresan de las aulas universitarias, se encuentran con una realidad llena de desigualdades y de pocas oportunidades para desarrollarse profesionalmente, y su futuro, supuestamente más certero en comparación a los jóvenes con menos oportunidades, se desdibuja; incluso llega a reducirse a planes y trayectorias de vida a corto plazo.

El objetivo del presente trabajo es analizar cómo jóvenes egresados de las áreas de las ciencias sociales, de la universidad, una pública y otra privada, del centro del México, específicamente el Estado de México, están significando y construyendo sus sentidos sociales frente a la violencia estructural que se vive en la realidad nacional. El estado de México es considerado, según los Indicadores de Marginación Absoluta de las Entidades Federativas 2000-2010 (CONAPO, 2010) como uno de los estados que presenta una baja marginación a diferencia de otros estados como Guerrero, Oaxaca o Chiapas.

Para dar respuesta a dicho objetivo, el trabajo se divide en tres grandes apartados, en el primero de ellos, se hará una revisión del concepto de 
violencia estructural, con algunos datos que marcan un panorama de la falta de oportunidades para los jóvenes en México. En el segundo, se aborda cómo desde los estudios de culturas juveniles podría plantearse el estudio de los jóvenes y la violencia estructural para la comprensión de los significados y respuestas que se hace de esta última. En uno tercero, se plantea un análisis cualitativo a egresados del área de ciencias sociales de una universidad pública del centro del país, de la significación de la violencia estructural y las maneras de hacerle frente, así como las expectativas del futuro que vislumbran. Finalmente se plantean algunas conclusiones generales, que dejan entrever las frustraciones y contradicciones que les permea la violencia estructural y cómo, ante la misma, los jóvenes, en su cotidianeidad, se reinventan estrategias para hacerle frente.

\section{VIOLENCIA ESTRUCTURAL Y JÓVENES}

Galtung considera «que la violencia está presente cuando los seres humanos se ven influidos de tal manera que sus realizaciones efectivas, somáticas y mentales, están por debajo de sus realizaciones potenciales» (Galtung, 1995:314). El espectro de violencia aparecería, por tanto, cuando por motivos ajenos a nuestra voluntad, no somos lo que podríamos ser o no tenemos lo que podríamos tener.

Entender la violencia, desde dicha perspectiva, presupone tres elementos a considerar: alguien que influye (sujeto), la influencia (objeto) y el modo de influir (acción); elementos que Galtung (1995) retoma para plantear algunas distinciones y así entender la acción violenta. Derivado de ello, se puede plantear una tipología de la violencia: la personal o directa (visible porque se manifiesta en los golpes, gritos, insultos, etc.); la estructural o indirecta (se encuentra en las estructuras sociales), y la cultural (arraigada en la cultura y que legitima cualquier otro tipo de violencia).

Particularmente en la violencia estructural, no se identifica al emisor de la misma, o a una persona concreta que haya efectuado el acto de violencia; se caracteriza porque «está edificada dentro de la estructura y se manifiesta como un poder desigual y, consiguientemente, como oportunidades de vida distinta» (Galtung, 1995: 320).

Cuando las estructuras sociales no permiten a ciertos grupos sociales tener acceso lícito a los medios para cubrir sus necesidades, estamos ante la presencia de violencia estructural, evidentemente relacionada con la injusticia social. Entre las manifestaciones de la violencia estructural se 
encuentran: la exclusión (no-participación), desigualdad, pobreza y la falta de oportunidad de acceder a bienes como pueden ser alimentos, drenaje y agua potable, vivienda, educación, atención médica, entre otros; pero también, la represión de clases antagónicas, violación de derechos, alineación cultural, concentración del poder político, falta de democracia, etc.

Este tipo de violencia, se encuentra oculta en las estructuras sociales, políticas y económicas de las sociedades, haciéndose evidente cuando existe incapacidad por parte de los gobiernos para cubrir las necesidades básicas de su población, no se respetan los derechos humanos fundamentales, o no se permite que la ciudadanía se exprese o participe libremente. Frecuentemente la presencia de violencia estructural no es percibida como tal o es invisibilizada por la violencia cultural, ${ }^{1} \mathrm{e}$ incluso logra hacerla parecer como algo natural y/o inevitable.

Matices de la violencia estructural en las juventudes en México

\section{Educación, empleo y jóvenes}

En México el derecho a la educación superior pública de gran número de jóvenes se ha coartado, el ejemplo más evidente es el de la universidad más grande del país, la Universidad Nacional Autónoma de México (UNAM), la cual ofrece más de cien licenciaturas, en tres modalidades educativas: escolarizada, abierta y a distancia. Para el año 2013 aplicaron para el Primer Examen de selección a nivel licenciatura 126.753 jóvenes, de los cuales sólo el 8,6 por ciento (10 mil 916 jóvenes) fueron seleccionados, es decir alrededor del 92 por ciento de quienes aspiraban a cursar estudios superiores en esa institución, fueron rechazados. Derivado de las marchas y movilizaciones que en protesta realizaron los jóvenes, se convocó a un segundo examen de ingreso, no obstante solo fueron seleccionados el 12,8 por ciento (7 mil 923 personas) de quienes aplicaron el examen, esta situación se reprodujo en cada una de las instituciones públicas de educación superior del país (La Jornada, 11 de abril y 15 de julio de 2013).

En este sentido, la Organización para la Cooperación y el Desarrollo Económicos (OCDE) en su Panorama Educativo 2013, afirma que en México

1 Para Galtung, la violencia cultural son razonamientos, actitudes, ideas que promueven, legitiman y justifican la violencia en su forma directa o estructural, así la cultura puede conducir a ver la explotación y/o la represión como hechos normales y naturales, o no verlos como tales. 
la mayoría de la población, 64 por ciento, cuenta con un nivel de educación inferior al bachillerato. Por su parte la Encuesta Nacional de Valores en Juventud (ENVJ 2012), ${ }^{2}$ al preguntar a los jóvenes si alguna vez estudiaron, el $97,3 \%$ respondieron afirmativamente, es decir la mayor parte de ellos alguna vez tuvo acceso a la educación; sin embargo, la mayoría de los encuestados, el 51,8 \%, solo ha recibido la educación básica, el 28,2 por ciento ha cursado la escuela preparatoria o bachillerato, el 10,5 por ciento tiene estudios profesionales, mientras que el $7 \%$ de los entrevistados estudio una carrera técnica.

Por otra parte el 53,5\% de los jóvenes encuestados consideran que la educación es el medio para obtener un buen trabajo, para el 9,3 por ciento la educación se traduce en un mecanismo para ganar dinero, mientras que el $8 \%$ considera que su propósito es obtener conocimientos y el 1,2\% le permite tener prestigio personal. Sin importar la motivación que los jóvenes tienen para estudiar, el 93,9\% de los jóvenes que participaron en la encuesta consideran que vale la pena estudiar una carrera profesional.

Sin embargo, en México las estadísticas nos muestran que hoy en día no se puede asociar la preparación profesional con la inserción laboral, tampoco garantiza mayor movilidad ascendente, ni una retribución económica adecuada. Para la Encuesta Nacional de la Juventud 2005, los jóvenes de 14 a 29 años que solo estudiaban representan el 43,7 por ciento, pero para el año 2010, dicho porcentaje disminuyó en 11 puntos, ya que solo el 32,5\% estudiaba. De igual forma, se observa que en el 2005 los jóvenes que estudiaban y trabajaban era del 5,3\% y para el 2010 se incrementó al 11,2 \%; es decir, se incrementó en casi 6 puntos. Dichos datos, nos muestran que un mayor número de jóvenes tiene la necesidad de trabajar, no solo para continuar con sus estudios, sino también para aportar al ingreso familiar.

En este sentido Loria Díaz, investigador del área de economía de la UNAM, afirma que en México los jóvenes en el rango de 14 a 24 años de origen pobre, cuyo nivel educativo es básico y no cuentan con experiencia laboral logran emplearse en mayor número que los jóvenes que provienen de clases medias o altas que cuentan con educación media-superior o superior. Así en este país, los jóvenes con mayor preparación académica tienen mayor probabilidad de encontrarse desempleados. Lo cual, explica el académico, obedece a que los jóvenes de 14 a 24 años de extracción pobre, con

2 La Encuesta Nacional de Valores en Juventud 2012, fue realizada por Instituto Mexicano de la Juventud (Imjuve) y el Instituto de Investigaciones Jurídicas de la unAM. Su metodología consistió en un muestreo probabilístico, estratificado, polietápico y por conglomerados. La muestra se integró por 5000 jóvenes de los ámbitos rural y urbano de toda la República Mexicana y fue levantada durante el mes de septiembre del año 2012 (Imjuve, 2012). 
escaso nivel académico están dispuestos a aceptar empleos con bajos sueldos, mientras que los jóvenes de nivel socioeconómico alto, con estudios superiores y experiencia laboral, esperan recibir un salario mayor (La Jornada, 21 de mayo de 2014:14).

Así, la investigadora Fiorella Mancini, afirma que en México es poco probable que un joven ingrese en condiciones adecuadas al mercado laboral debido al incremento del trabajo informal, el detrimento de las condiciones laborales, los bajos salarios, y la falta de prestaciones. Las estadísticas muestran que el 66 por ciento de los jóvenes mexicanos en un rango de edad entre 15 y 29 años está empleado en el mercado informal, lo cual se traduce en vulnerabilidad para ellos (La Jornada 20 de mayo de 2014:35).

A pesar de la realidad laboral de los jóvenes, la Encuesta Nacional de Juventud 2010 (ImJUVE, 2010), evidencia que sus expectativas respecto a un empleo es que sea bien remunerado (el 84,9 por ciento de los encuestados), que sea un trabajo estable $(42,6 \%)$ y con prestaciones $(40,4 \%)$.Asimismo el tener éxito para estos jóvenes se traduce en obtener un trabajo, y a través del mismo lograr un buen nivel de vida, tener educación, formar una familia y lograr las metas que se propongan alcanzar. Desde su perspectiva, los tres principales conflictos en México son la pobreza, el desempleo y la inseguridad.

Por su parte Oliveira (2008) analiza la intensidad y temporalidad de los eventos de transición a la vida adulta en jóvenes mexicanos: la salida de la escuela, la entrada al mundo laboral, la salida de la casa de los padres, la primera relación sexual, la primera unión, el primer embarazo y el nacimiento del primer hijo, considerando su estrato social (estratos medio-alto y bajo), género, así como la edad. Dentro de los resultados que nos presenta se encuentran que la inserción laboral es el primer evento de transición a la vida adulta para la tercera parte de los jóvenes de 15 a 29 años. Las y los jóvenes del estrato medio-alto presentan como primera transición la incorporación al mundo laboral, mientras que para las y los jóvenes del estrato bajo lo es dejar la escuela y en segundo lugar entrar a trabajar, lo que a lo largo del tiempo suele manifestarse como una desventaja para emplearse con mejores condiciones laborales.

De igual forma, en dicho estudio (Oliveira, 2008), se destacan contrastes importantes entre estratos sociales, así los hombres jóvenes del estrato bajo inician su vida laboral o dejan la escuela en mayor medida que el estrato medio-alto. Entre los motivos por los cuales dejaron de estudiar los jóvenes de 15 a 19 años, se encuentra el hecho de tener que trabajar, así, por ejemplo, cerca del 12 por ciento de las mujeres jóvenes del estrato 
bajo afirmó que tenían que trabajar y 7 por ciento que tenían que ayudar en su casa. Entre los hombres del mismo estrato social en un 27,2 por ciento el motivo fue el trabajo y el 3,8 por ciento ayudar en su casa, mientras que en el estrato social medio-alto el 8.5 de las jóvenes y el 6,7 por ciento de los hombres afirmaron que tenían que trabajar y ninguno mencionó el trabajo en casa como una razón.

La entrada a los mercados laborales tiene ciertos contrastes, de acuerdo al estudio de Oliveira (2008), se muestra que en los hogares con menos recursos económicos, los y las jóvenes muestran un mayor riesgo de ingresar a trabajar o dejar la escuela a edades más tempranas que en el estrato medio-alto. Los hombres del estrato bajo presentan una mayor probabilidad de iniciar su vida laboral a los 18 años de edad; en el estrato medioalto esto sucede a los 24 años. En el caso de las mujeres la mayor probabilidad de ingresar al mercado de trabajo es entre los 16 y 18 años en el estrato bajo y a los 22 años en el medio alto (cfr. Oliveira, 2008).

Los jóvenes sufren desigualdades que son notorias en sus accesos a la educación, el empleo y en los procesos de transición a la adultez. Ello es notorio en la interrupción de estudios y la entrada al mercado laboral, así como en la manera en que ellos permanecen en la casa paterna y no logran su independencia.

\section{Migración, delincuencia y juventud}

La vulnerabilidad laboral en la que se encuentran los jóvenes ocasiona que abandonen sus empleos y la migración se convierta en una posibilidad de encontrar mejores oportunidades laborales y escolares. La población joven que migra dentro del mismo país, en su mayoría cambia su residencia del ámbito rural al urbano, las zonas metropolitanas y las ciudades de mayor tamaño son las de mayor atracción de migrantes.

Los datos de la muestra censal de 2010 estiman que del total de personas de 15 a 29 años, 3,7\% cambió su municipio de residencia dentro de la misma entidad. Su estructura por sexo muestra una mayor participación de las mujeres en la movilidad municipal ya que hay 78 hombres jóvenes migrantes intraestatales por cada 100 mujeres. Si al porcentaje de jóvenes que se fueron a vivir a otro municipio dentro de la entidad se añaden los que cambiaron de entidad de residencia (4,3\%), entonces, ocho de cada cien jóvenes cruzó entre 2005 y 2010 algún límite municipal dentro o fuera de la misma entidad, para cambiar su lugar de residencia (INEGI, 2012). 
La proximidad con los Estados Unidos de Norteamérica y la creencia del llamado sueño americano permite una fuerte incidencia en la migración internacional, la cual es predominantemente masculina. En este sentido para «el periodo 2005-2010,57,1\% del total de emigrantes internacionales captados en la Muestra del Censo de Población y Vivienda de 2010 tenía entre 15 y 29 años de edad en su salida más reciente del país, de estos tres de cada cuatro son hombres. Los principales lugares de destino son Estados Unidos de América (91,4\%), Canadá (2,7\%) y España (1.1 por ciento) (INEGI, 2012).

La Encuesta de la Comunidad Americana 2010 muestra que del número de migrantes residentes en los Estados Unidos de Norteamérica, casi 12 millones son mexicanos y una cuarta parte de ellos $(25,3 \%)$ eran jóvenes en un rango de edad de 15 a 29 años, y en su mayoría eran varones (132 hombres por cada 100 mujeres) (citado en INEGI, 2012).

Es evidente que la principal motivación de estos jóvenes migrantes mexicanos que residen en ese país, es la laboral, así el 60,1\% de ellos desempeñó alguna actividad económica; y únicamente el 20,5\% estaba estudiando en su mayoría del 6 al 12 grado según el sistema escolar estadounidense. En su mayoría el 91,5\% de estos jóvenes viven con un familiar, 1\% vive solo y el 7,5\% convive en hogares donde no hay relación de parentesco con el responsable de la vivienda (cfr. INEGI, 2012).

Ciertas manifestaciones de violencia estructural (desempleo y falta de oportunidades de estudio), suelen traducirse en violencia directa, en este sentido para algunos jóvenes la delincuencia se ha convertido en una opción para su subsistencia, así las estadísticas en México sobre los delitos que se procesaron penalmente durante el año 2010 (cfr. INEGI, 2012).

«registraron 199 mil 812 personas a quienes el juez de primera instancia les dictó Auto de Término Constitucional relativo a los delitos por los que fueron consignados; en ese mismo año, 156 mil 401 procesados recibieron sentencia. Casi la mitad de los procesados (46,5\%) y de los sentenciados (48,6\%) son jóvenes de 18 a 29 años de edad. Cabe señalar que nueve de cada diez $(92,4 \%)$ jóvenes procesados o sentenciados son hombres» (cfr. INEGI, 2012).

Los principales delitos cometidos por los jóvenes sentenciados en esos juzgados del fuero común son en su mayoría «robo $(56,5 \%)$, golpes y lesiones $(13,9 \%)$, homicidio $(4,7 \%)$ y daños a propiedad ajena $(4,5 \%)$, en tanto que $62,2 \%$ de los delitos que recibieron sentencia en los juzgados del fuero federal fueron por narcóticos, $28,6 \%$ por actos ilícitos con armas, 1,7\% por tráfico de indocumentados y 1,1\% por robo» (cfr. INEGI, 2012). 


\section{JUVENTUDES, UNA APROXIMACIÓN A SU ESTUDIO}

Se parte del supuesto de que hoy en día no se puede hablar del concepto de juventud, sino más bien tenemos que referirnos al de juventudes, en tanto este nos va a permitir mirar epistemológicamente la diversidad y heterogeneidad de ese mundo social que se ve atravesado, por ejemplo, por la clase social que define la posición que ocupan en la estructura social.

Estudiar a las juventudes y no así a la juventud (homogénea), permite reconocer que éstas son portadoras de diferencias y singularidades que construyen su pluralidad y diversidad en los distintos espacios sociales; se recoge la riqueza de su pluralidad; se asume una vinculación directa e íntima con el mundo juvenil, múltiple y plural, como condición de la generación de conocimiento comprensivo del mismo en una realidad latinoamericana, y por último, se rompe con la rigidez de la mirada mecanicista con la que se ha mirado y hablado de la juventud. Desde dichas pistas se podría visibilizar a las juventudes no solo desde quien las nombra, sino desde ellas mismas, quienes «van ganando historicidad desde sus propias expresiones y muchas veces irrupciones en el espacio social» (Duarte, 2000: 76).

Por otra parte, el concepto de «jóvenes» es una categoría sociocultural, producto de la posguerra ; siendo la dimensión cultural central en la constitución de la comprensión de los jóvenes; aunque fueron los acontecimientos políticos (el desgaste del sistema, la crítica al autoritarismo y la respuesta represiva a las manifestaciones juveniles, entre otros factores) los que organizaron y orientaron, en buena medida, la comprensión en torno a la categoría jóvenes (Reguillo, 2010: 10). Por ello mismo, para la autora, los jóvenes se volvieron visibles gracias a sus protagonismos en el espacio público.

La configuración de identidades múltiples y cambiantes que articulan los gustos sexuales, el consumo cultural y los espacios de socialidad y de amistad son puntos nodales para la comprensión de lo juvenil (Rodríguez en Ramírez, 2010: 354).

Este paradigma que plantea a los jóvenes como construcción sociocultural se propone como un espacio teórico o interpretativo que ayuda a comprender la construcción de la juventud como institución social, más allá de la actividad de cualquier joven en particular. Algunas características de este paradigma son: 
1) La importancia de concebir la juventud como construcción social de una fase particular en el ciclo de vida que cambia de forma y de contenido a través del tiempo y del espacio.

2) La juventud como una variable del análisis social no puede ser separada de otras variables, como género, clase, étnica, región, entre otras.

3) La juventud, las relaciones sociales y las culturales de los jóvenes merecen ser estudiadas en sus propios términos; esto es, sin perder de vista los aspectos más relevantes y particulares de sus vidas, aquellos que ellos mismos ponen por delante (Urteaga, 2010: 18-19).

La autora plantea que es necesario ver cómo los actores juveniles se están estructurando y reconfigurando, a través de sus prácticas sociales y culturales, y representaciones sobre las mismas, nuevas realidades y significados de clase, género, etnia, y cómo estas, reconfiguradas, estructuran la condición juvenil contemporánea (Urteaga, 2010).Así, por ejemplo, una de las prácticas sociales que es eje fundamental para su constitución como sujetos sociales, es la espacialización pública de su sociabilidad, en tanto esta interviene/ transforma / configura la ciudad, al tejer y articular fronteras de la diferencia entre los grupos juveniles que participan en contextos locales urbanos y globales metropolitanos comunes o convergentes (Urteaga, 2010: 27).

La condición juvenil, entonces, se entiende como el conjunto multidimensional de formas particulares, diferenciadas y culturalmente «acordadas» que otorgan, definen, marcan, establecen límites y parámetros a la experiencia subjetiva y social de las/los jóvenes (Reguillo, 2010: 41). La condición juvenil no es unívoca y es siempre el resultado de la articulación compleja de múltiples factores, en los que la cuestión de clase y género constituyen factores o estructurantes de esta condición.

Las categorías «juventud», «clase», «etnia» y "género» no son neutras, pues conforman tipos específicos de desigualdades, producto de relaciones sociales y de poder históricamente constituidas en cada país y región. Cada categoría (juventud, clase, etnia y género) tiene diferentes ámbitos o niveles de análisis: un aspecto microsocial (vinculado con enfoques que subrayan las capacidades individuales), un aspecto relacional o interaccional (vinculado con la producción de estas desigualdades en diversos campos de la interacción social) y un aspecto estructural (más holístico, ubicado en las estructuras de posiciones y mecanismos más amplios de distribución de los beneficios y las cargas) ( Reguillo, 2010: 15- 16). 
En México, en los últimos 20 años, son tres los tipos de capital ${ }^{3}$ elusivo para una inmensa mayoría de los jóvenes en el país:

a) El capital cognitivo-escolar y de destrezas, valorable en términos de mercados y de redes.

b) El capital social, cuyo valor estriba en su potencial para solventar las carencias del individuo y cuya argamasa se sustenta en su flexibilidad y pertinencia en términos, otra vez, de mercado.

c) El capital político, cuya importancia estriba en la posibilidad de intercambiar posición (objetiva) por reconocimiento (simbólico) (Reguillo, 2010: 397).

A partir de dicha tipología, se reconoce que el capital objetivo carece de relevancia si no es acompañado del social y el político en el contexto de la atmósfera neoliberal que existe en el país. Es decir, los grados escolares, las destrezas que puedan adquirirse, carecen de sentido si no van acompañados de relaciones sociales y lógicas de reconocimiento social que «avalen» o sustenten las destrezas objetivas (Reguillo, 2010: 397-398). El capital más importante para los jóvenes rurales, o más empobrecidos de las zonas urbanas y semiurbanas se sustenta en la familia y en las relaciones de amistad; mientras que entre el conjunto de jóvenes urbanos y de sectores medios y altos, este capital se vincula con las instituciones, la escuela, la empresa, el Estado.

Los jóvenes excluidos no se caracterizan por una ausencia radical de los capitales relacionales, culturales, económicos e institucionales, sino por una considerable debilidad de los mismos (Makowki, 2010). Así por ejemplo, los caracterizados como frágiles son aquellos que comienzan a experimentar un distanciamiento respecto del mercado laboral o dificultades para su acceso (como es el caso de los jóvenes), y están expuestos a las resocializaciones, negociaciones de la identidad, a la formación de estrategias de respuestas y a la construcción de racionalizaciones sobre su nueva situación (Frétigné, citado por Makowski, 2010: 46).

Estudios como los de Reguillo (2007, 2010), Urteaga (2010), Cisneros (2007), dejan ver que se tiende a culpabilizar a los jóvenes de la precariedad de sus vidas, y en muchas de las ocasiones se tiende a dejar la responsabilidad de su situación a ellos mismos; omitiendo la responsabilidad del Estado y de las instituciones públicas para dar respuesta a las necesidades

3 Reguillo, siguiendo a Bordieu, entiende por capital, los recursos tanto materiales como simbólicos a los que los actores tienen acceso al interior de las relaciones sociales. 
educativas y de salud que requieren.Así, los jóvenes pobres no solo sufren de violencia estructural, sino que además, se hace toda una construcción social que los coloca en una situación de mayor distanciamiento social. De ahí, que no hay mayor adversario para la agencia juvenil que su propia y fatalista asunción de «inadecuación» social, política y laboral (Reguillo, 2010: 399).

Frente a las crisis económicas, políticas, medioambientales y energéticas que vive el país, los jóvenes sufren desigualdades, entendiendo que pobreza no es lo mismo que desigualdad. Pobreza es la insatisfacción de las necesidades básicas y desigualdad es la distancia (Tortosa, 2012).

En ese sentido, existen «dos juventudes mexicanas»: una mayoritaria, precarizada, desconectada no solo de lo que se denomina la sociedad red o sociedad de la información, sino separada o desafiliada de las instituciones y sistemas de seguridad educativa, de salud y de trabajo; sobreviviendo apenas con los mínimos, y cuyos reclamos se inscriben en una lógica estructural, aspiraciones elementales de justicia social y bienestar. Pero, también está la otra juventud, la minoritaria, la «bien ubicada», la que tiene conectividad, la que se encuentra incorporada a los circuitos de seguridad y en condiciones de elegir, es decir tienen acceso a satisfactores elementales como educación, empleo y salud (Reguillo, 2007; 2010).

Quizá en el plano de lo estructural, se puede afirmar que el núcleo de desigualdad entre las/los jóvenes mexicanos se concentra en dos palabras clave: alternativas y acceso. De un lado la posibilidad de elegir, de optar o no, de organizar o no, una ruta biográfica en la que sea posible, o no, acceder a los espacios, instituciones, sistemas que ofrezcan un mínimo de certezas para imaginar el futuro; siendo tres las instancias clave que están hoy operando como espacios para la «reinscripción»o «reapropiación» del yo juvenil: 1) las estructuras del crimen organizado o narcotráfico, 2) la diversidad de ofertas y ofertadores de sentido, y 3) el mercado a través de sus ofertas de identidad (Reguillo, 2010: 396).

Para los fines del presente trabajo, el imaginar el futuro juega un papel fundamental, porque es una de las maneras que tienen los jóvenes para hacerle frente a esa inseguridad provocada por la desigualdad. Oliveria (2014) afirma que en el estudio de los jóvenes y sus sentidos de vida, se tiene que abordar el concepto de temporalidad; es decir, son sujetos que tienen pasado, presente y futuro. Dependiendo de ello, se pueden visualizar las maneras en cómo pueden construir trayectoria lineales (cuando el futuro se tiene imaginado) o bien sus trayectorias son forzadas por las condiciones materiales de vida «coyunturales» de tal suerte que su futuro ima- 
ginado se va acortando. Planteamiento que se amalgama con los conceptos de «alternativas y acceso» como estrategias para hacerle frente a la desigualdad.

Los jóvenes, frente a dichas desigualdades, han ido encontrando estrategias alternativas tanto en el empleo como en su propia autonomía; una de ellas es concluir antes los estudios, con las facilidades que brindan muchas escuelas; otra, es abandonar los estudios e incorporarse al mundo laboral. Vale la pena decir que, en una generalidad, aquellos jóvenes que logran vivir de manera independiente de sus familias, van retrasando la construcción de una familia propia. Parece que el joven no cuestiona las pautas de tránsito hacia la edad adulta, basada en las responsabilidades, sino que opta por reducir sus expectativas y disfrutar las cosas que se le van presentando, gratificaciones razonables respecto a su identidad. Sin embargo, hay que reconocer que las trampas de la desigualdad son muchas y sus capitales y agencias no son suficientes (cfr. Urteaga, 2010; Reguillo, 2010).

\section{LOS JÓVENES EGRESADOS: SUS ESTRATEGIAS Y MIRADAS ANTE EL FUTURO}

Explicaciones metodológicas

El presente apartado pretende mostrar resultados del análisis de los significados y sentidos sociales que hacen jóvenes, egresados de licenciatura de las áreas de las ciencias sociales, frente a la violencia estructural que se vive en la realidad nacional y cómo, con ello, dibujan su futuro. Bajo la acotación de que son estudiantes de dos universidades ubicadas en el Estado de México; estado del centro del país, el cual es considerado uno de los estados con mayor desarrollo económico a nivel nacional.

La razón de seleccionar a egresados de licenciaturas en ciencias sociales (sociología y comunicación) obedeció principalmente a dos motivos: el primero de ellos tiene que ver con las competencias de análisis de la realidad social que permea su formación; el segundo, el abanico de alternativas de inserción laboral ${ }^{4}$.

4 Para mayor información sobre competencias profesionales y mercados profesionales de los egresados de sociología y comunicación, de la Universidad Autónoma del Estado de México, ver uAEm (2004) Licenciatura en comunicación. Curriculum 2004, facultad de ciencias políticas y administración pública: Toluca y UAEM (2004) licenciatura en sociología. Curriculum 2004, facultad de ciencias políticas y administración pública:Toluca. 
El estado de México cuenta con una universidad pública, la Universidad del Estado de México (UAEM); cuya rectoría se encuentra en la capital del Estado de México (Toluca). Además, hay licenciaturas que se ofertan en universidades privadas, pero que tienen el respaldo de la Universidad Autónoma del Estado de México a razón de llevar el mismo programa y plan de estudios; a dichas licenciaturas se les denomina como incorporadas a la UAEM. Para el caso de comunicación, hay varias universidades que ofertan la licenciatura en comunicación incorporada a la UAEM. Vale la pena aclarar que los egresados de la UAEM o escuelas incorporadas, necesitan presentar un trabajo terminal, examen general, o haber sido alumnos sobresalientes, para acceder al título de licenciados.

En este sentido, se buscó que hubiera egresados de la UAEM $^{5}$ y algunos otros de la universidad incorporada ${ }^{6}$. La elección de dichas universidades nos permite contar con sujetos que cuentan con una formación común, pero con contextos diferentes; ello amplía el panorama sobre la construcción de sentidos y significaciones que se tiene frente a las desigualdades y las trayectorias de vida. Dicha elección metodológica expresa que, si bien son sujetos considerados como «integrados» ${ }^{7}$, también es cierto que sus capitales y agencias son distintas $y$, por ende, sus estrategias frente a la desigualdad son diversas, dependiendo de sus alternativas y accesos.

En función de lo anterior, se determinó ocupar técnicas de corte cualitativo que pudieran ayudar a resolver el objetivo planteado. Se decidió ocupar un grupo focal ${ }^{8}$, y para el análisis del corpus discursivo, derivado

5 Los estudiantes de la UAEM, provienen de diversos municipios, incluso, de otras entidades federativas; y tienen acceso a una serie de becas que les permiten resolver una parte económica importante para el desarrollo de su vida académica.

6 La universidad incorporada se ubica en una zona cercana a la capital del Estado (Toluca); en un municipio con población mayoritariamente de ascendencia indígena (mazahua). Entre las características de la población estudiantil de esta última universidad, se encuentran que el estudiantado proviene de distintos municipios cercanos, incluyendo Toluca, pero rara vez de otra entidad federativa; estudian ahí porque no tuvieron la oportunidad de ingresar a la UAEM cuentan con poco acceso a becas o estas son muy limitadas; y en la mayoría de los casos provienen de familias con recursos económicos limitados (por lo que hacen un gran esfuerzo para pagar las colegiaturas).

7 Definición planteada por Reguillo $(2007,2010)$, y que aquí se recupera, para plantear que los egresados son sujetos «integrados» por el simple hecho de haber estudiado una licenciatura y haber asistido a la universidad.

8 El grupo focal es considerado un dispositivo de investigación sociológica en el cual se hace evidente la manera en que los sujetos y los grupos construyen y dan sentido a los acontecimientos y circunstancias en que viven, se hace emerger las categorías e interpretaciones que se generan en los marcos intersubjetivos de la interacción social, por medio de procesos comunicativos y lingüísticos. A través de la discusión grupal, se suscitan las representaciones simbólicas sensibles y sociales del grupo de referencia (Alonso, 1998). 
de ello, el análisis crítico del discurso 9 . Justamente, porque el grupo focal es un juego del lenguaje colectivo que produce un discurso, el cual servirá de materia prima para el análisis.

La discusión oral que se produce en el grupo focal se transcribe a una versión estenográfica, de tal suerte que se convierte en el corpus discursivo objeto de análisis. Discurso, en donde se observa la construcción significativa del mundo, en este caso que hacen los egresados, a partir de su experiencia.

Para conformar el grupo focal se consideró lo siguiente: tendrían que ser egresados de las licenciaturas en comunicación, ciencias políticas y/o sociología, de cualquiera de estas dos universidades ${ }^{10}$; el tiempo de egreso tendría que ser menor a tres años (un tiempo mayor de egreso podría llevarnos a que los sujetos tuvieran una vida con todas las seguridades cubiertas y con una mayor estabilidad económica y profesional); podían o no estar titulados.

Los contactos personales de las investigadoras, permitió reunir a los miembros; pero para evitar que se conocieran previamente entre sí, se buscaron que fueran de generaciones o grupos distintos. El grupo quedó conformado de la siguiente manera:

9 Este estudia el lenguaje como práctica social y considera que el contexto de uso es crucial, se interesa, además, por la relación entre el lenguaje y el poder.

10 Aunque se solicitó el apoyo de egresados de licenciaturas en sociología y administración pública, asistieron mayoritariamente de la licenciatura en comunicación. 


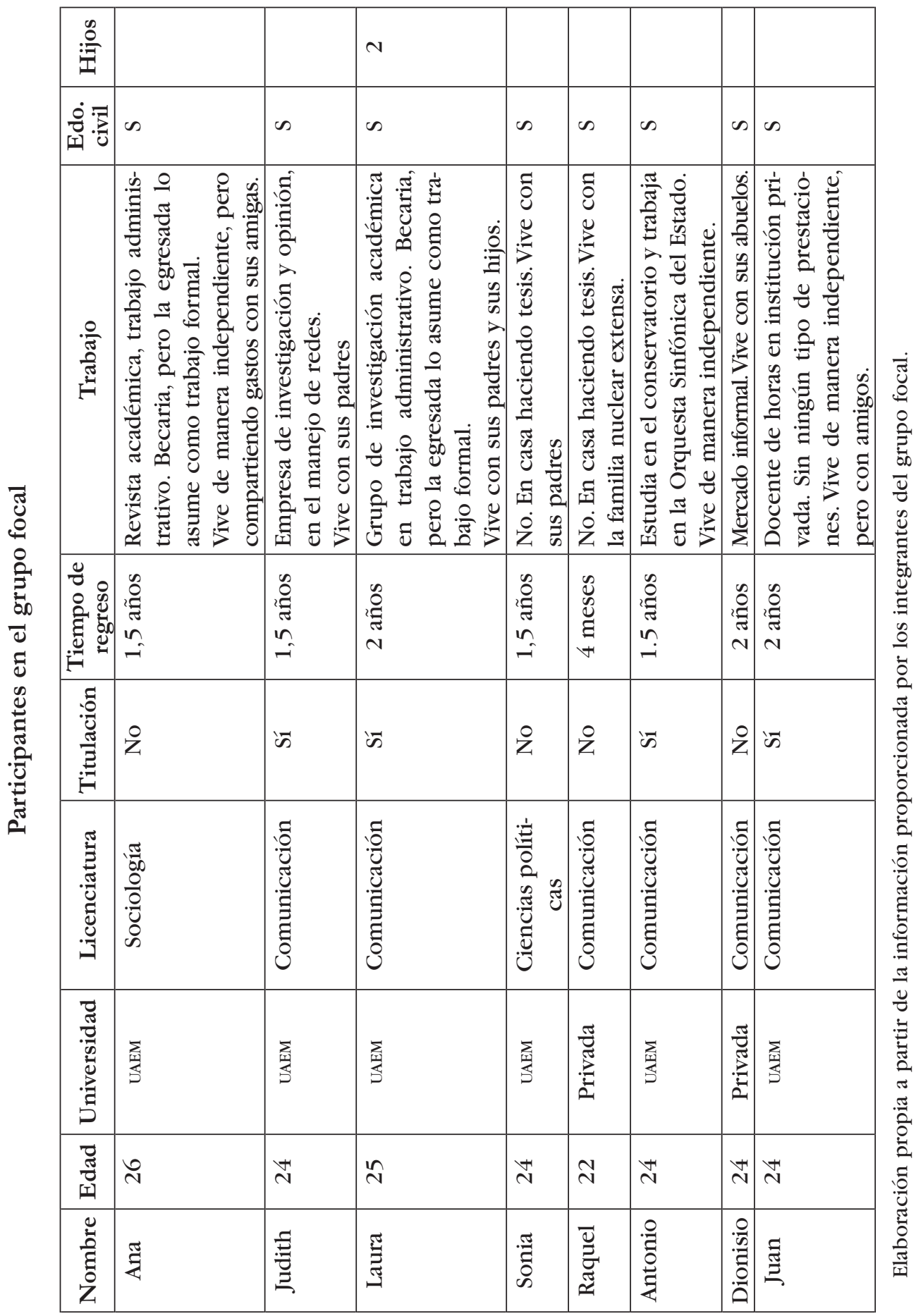


Los ejes que guiaron el grupo focal se centran en tres tópicos: sus recursos y capitales, su mirada hacia la universidad y el futuro que visualizan, bajo los cuales se presentan los siguientes resultados.

Quiénes son, qué hacen y cuáles son sus capitales

En el grupo focal se rescatan algunas evidencias de la violencia estructural que sufren los jóvenes participantes en materia de lo laboral, de los ocho participantes, cinco afirmaron estar empleados, pero solo dos de ellos lo hacen de manera formal, aunque ambos carecen de prestaciones laborales. Las restantes tres personas reciben becas o pagos simbólicos por la actividad que desempeñan:

[...] es mucho trabajo, aunque sea como becaria, pero, pues el salario es muy poco, muy muy poco, entonces sí es muy necesario comenzar la búsqueda de un trabajo o un complemente a esto (Ana).

Varios de ellos manifestaron la dificultad para encontrar un empleo y declararon cómo a pesar de contar con trabajo, este no cumple sus expectativas; sin embargo, no se les ha presentado otras opciones. Se visualiza que encontrar trabajo pareciera una cuestión coyuntural y/o de suerte. Por ello mismo, la definen como oportunidad.

Yo quisiera encontrar un trabajo, ahorita es muy difícil si no tienes un título y aunque tengas los estudios terminados y tengas un perfil o un este, o cierta experiencia (Sonia).

[...] eso mismo me orilló pus a trabajar en el comercio informal y pus sigo en la lucha incesante de la búsqueda de trabajo, pero también sé que algún día lo voy a lograr y que lo tengo que conseguir (Dionisio).

Igual para mí no fue como tan fuerte el cambio, eeh , porque también tuve la oportunidad de trabajar mientras estudiaba, algunos meses...prácticamente terminé la escuela y afortunadamente pues se abrió una puerta en esta empresa (Judith).

La independencia del núcleo familiar es una preocupación generalizada a pesar de que solo tres de los jóvenes ya no viven con sus padres. La independencia la lograron justo porque tuvieron que migrar a otra ciudad para poder continuar sus estudios; ${ }^{11}$ actualmente ya han logrado indepen-

11 En el estudio de Oliveira (2008), se encuentra que las y los jóvenes del estrato bajo apresuran su salida del hogar de su padres en comparación con los del estrato medio-alto. Las desigualdades sociales se hacen evidentes al conocer las razones para dejar la casa por primera ocasión entre los jóvenes de 15 
dizarse económicamente de sus familias, pero no por ello se encuentran necesariamente en una posición estable, más bien tienen estrategias para sobrevivir, como lo es rentar con amigos para cubrir los gastos.

[...] desde los 11 años dejé de vivir con mis papás, después comencé a vivir con una prima y ella pues me apoyaba y yo la apoyaba a ella (Ana).

Ahorita comparto un departamento con, con unas chicas que conocí en un sitio de rentas por internet, entonces... llevo como 5 años viviendo fuera de mi casa, entonces, yo creo que eso de vivir es estar en el lugar y permanecer en el lugar, pero en realidad tengo como 8 años fuera de mi casa, entonces, ahora, comparto este departamento y tengo que solventar junto con las chicas pues los gastos de la renta, ¿no? De los servicios, ¿no? Luz, agua, teléfono (Juan).

La independencia del hogar familiar es una de las metas que tienen algunos de los otros cinco estudiantes, pero no han logrado realizarla por cuestiones económicas; en uno de los casos la participante contrajo matrimonio y vivió con su esposo, al momento del divorcio nuevamente vivió con sus padres.

[...] en realidad ya planeaba no vivir con mis papás, planeaba ser más autosuficiente, pero por algunas cosas en mi vida, no he podido (Raquel).

Yo vivo con mis papás, con mi hermano, bueno mi hermana está divorciado y ahora relativamente también (Judith).

[...] pero a mis papás se les ocurrió compartir los gastos y también me ayudaron mucho, entonces ellos ya se fueron a vivir conmigo, actualmente compartimos los gastos de la casa y los míos y de mis pequeños yo los cubro y... pues sí, esa es la manera, ahorita vivimos mi papá, mi hermano, mi mamá, mis dos pequeños y yo (Laura).

La solvencia económica juega un papel fundamental para lograr su independencia, los egresados se apropian de la ecuación de que por el simple hecho de contar con capitales cognitivos a nivel universitarios, podrían entrar con facilidad a las redes del mercado profesional; sin embargo, si éste no se ve vinculado con sus capitales sociales y políticos, no pueden moverse en los niveles que ellos quisieran. Por eso mismo, los asumen como cuestión de suerte, misma que se ve favorecida por el hecho de per-

\footnotetext{
a 19 años de edad, del estrato bajo, el principal motivo es salir a trabajar fuera de su lugar de origen (el 19,5 por ciento de las mujeres y el 39,1 de los hombres) o porque comienzan a vivir en pareja o se casan. Mientras el principal motivo entre los jóvenes del mismo rango de edad del estrato medioalto es estudiar en otra ciudad, estado o país (38,4 por ciento de las mujeres y 29,4 por ciento de los hombres) mientras los del estrato bajo lo hacen mayormente porque se van a trabajar fuera de la ciudad, estado o país (39,1 de los varones y 19,5 por ciento de las mujeres) o porque se unen o se casan.
} 
manecer en su ámbito laboral desde su condición de estudiantes, aunque este no sea necesariamente su ámbito profesional.

\begin{abstract}
Bueno sinceramente la cuestión afuera es muy diferente a lo que tenemos aquí adentro, ... creí que las cosas estaban más fácil para incursionarte en el trabajo y seguir con tus estudios, sin embargo no es tan así de sencillo...el trabajo en el que estaba no llenaba mis expectativa si no era como que a mí me gustara (Sonia).

Pues mi experiencia ha sido la verdad es que grata... donde estoy trabajando es el lugar donde inicie mis prácticas profesionales, entonces me dieron la oportunidad de quedarme y me siento ahorita (Laura).
\end{abstract}

Las condiciones estructurales que enmarcan las condiciones de vida de los egresados, de cierta manera, están determinando si ellos pueden visualizarse en el mundo profesional. No solamente son cuestiones de pobreza, sino más bien, son producto de las desigualdades que están sufriendo.

\title{
Su mirada a la Universidad
}

La socialidad de los jóvenes es muy importante para construir las redes que les van ayudando a entrar al mundo laboral. Las instituciones, como la universidad, les permiten tener acceso a recursos que les ayudarían a moverse en la estructura social. La universidad les otorga un abanico de oportunidades para que ellos elijan, situación que les hace rechazar condiciones que legitimen cualquier tipo de violencia, y buscar la entrada a un desarrollo personal y profesional con un alto grado de satisfacción. Sin embargo, esto no es así para muchos de ellos:

De repente te sales, y sales de la escuela y tienes que buscar trabajo y de repente las oportunidades laborales son muy escasas y si a eso le agregamos que no estaba titulado [...] eso mismo me orilló pus a trabajar en el comercio informal y pus sigo en la lucha incesante de la búsqueda de trabajo, pero también sé que algún día lo voy a lograr y que lo tengo que conseguir (Dionisio).

Se puede observar cómo la Universidad, les otorga los capitales cognitivos, aunque no los sociales y políticos. Existen factores interrelacionados que confluyen para que los jóvenes puedan adquirirlos, tales como sus propios procesos de empoderamiento que traen del contexto (capacidad de elección e independencia, por ejemplo) aunados a los factores de riesgo en los que se desarrollan, así como sus propias condiciones materiales de vida (o la violencia estructural que sufren). Factores mismos que les darían competencias, por una parte, a la entrada del mercado profesional, por 
otra, para encarar las desigualdades a las que se enfrentan. A partir de dichos capitales y factores, construyen una visión de su paso por la Universidad:

lo que más extraño es como la vida estudiantil, esta vida cómoda, esta vida de becas, esta vida de socializar todo el tiempo... mi formación como fue muy muy distinta a la de mis otros compañeros, bueno yo tuve la oportunidad de estar de movilidad, ... estaba metido en un montón de cosas, , ... ahora he encontrado un trabajo de verdad, suena como muy, como muy este, vaya como una burla porque en realidad ya no tenemos trabajo de verdad, hay trabajos, pero ya no hay trabajos en los cuales, te permites desarrollarte profesionalmente... no hay una paga que te motive... tomando en cuenta las oportunidades hoy vivimos más en un tiempo de relaciones públicas y de contactos, más que de experiencia profesional ¿no? (Juan).

Con dicha declaración se observa, que si bien la universidad le ayudó con algunos elementos cognitivos, su paso y su desempeño profesional y personal, lo debe más a los procesos de socialización y movilidad que se realizó en ella. De igual forma, la visión que se están formando de la universidad, como institución que les ayuda a adquirir y permanecer en un estado de «conectados» (en palabras de Reguillo), se está desfigurando; pues para ellos, la Universidad resulta insuficiente para las demandas laborales, no así las relaciones sociales:

es una cuestión de estarle poniendo empeño de verdad a lo que se hace, porque puede ser que sí tengamos un buen trabajo y que lo hayamos obtenido por cuestión de relaciones pero, por ejemplo yo donde estoy llegué ahí a hacer mi servicio social, me gustó, continué con mis prácticas y me ofrecieron la oportunidad para quedarme ahí (Ana).

Otro de los problemas que visualizan como limitante de encontrar un trabajo remunerado es la obtención del título. La conclusión del trabajo de tesis, para aquellos que no tienen el título, es fundamental para conseguir otros proyectos, ya sea seguir estudiando o encontrar un trabajo. La conclusión de la tesis se ve como una alternativa para tener acceso a otro tipo de recursos; pero también se plantea como sueño a corto plazo:

Yo no me he titulado, bueno entonces, como parte de mis proyectos es, concluir mi tesis, ... mi línea de investigación es línea de la salud, me interesa mucho eso, continuar aprendiendo a cerca de eso, y, estaba interesada por una maestría que es de educación inclusiva (Ana).

Bueno, uno de mis proyectos a corto plazo es ya poderme titular, porque la necesidad de mi título si ya es muy urgente, en cuestión de ya laborar (Sonia). 
Discursivamente asumen la existencia de la violencia estructural; se hace evidente cuando ellos tienen historias desvinculadas al momento de estudiar algo y trabajar en otra cosa, situación que es notoria para aquellos que de cierta manera sufren mayor desigualdad:

[...] desde pequeñito siempre me ha llamado mucho la atención el tema de la educación entonces me gustaría trabajar en algo parecido o relacionado a lo que es la educación (Dionisio).

También pienso hacer una maestría y planeo irme a vivir a Xalapa. Entonces estaba pensando con unos compañeros, crear como, crear como un colectivo en el cual vendiéramos como varias cositas... a largo plazo me gustaría dedicarme de lleno en la investigación (Raquel).

Contrariamente, los egresados que tienen mejores condiciones materiales de vida y sufren menos de desigualdad; aquellos que están más conectados, construyen trayectorias mucho más lineales, aún a pesar de tener alguna que otra situación difícil:

\footnotetext{
Bueno, pues actualmente estudio una especialidad, aquí en la facultad y pues espero ya terminarla concluirla, y después quisiera estudiar una maestría en antropología (Laura). Del lado profesional pues sí me gustaría obtener un trabajo en dirección creativa, más o menos. A largo plazo sí tengo plan de realizar una maestría, obviamente después de realizar estos proyectos, y, bueno va a ser una maestría en el extranjero y bueno va a ser en escritura creativa (Juan).
}

Los jóvenes tienen conciencia de la insuficiencia en las condiciones para su desarrollo; aunque no tienen claridad en las causas, ya que por una parte, asumen que la Universidad no les da las competencias necesarias, pero por otra, reconocen que las crisis económicas y políticas, están provocando una mayor desigualdad. Lo cierto es que apropian su condición de clase, vislumbrando la necesidad de proponer nuevos escenarios para entrar al mercado profesional, donde la Universidad, como institución, no les está brindando la seguridad.

Las crisis que se viven en la realidad nacional han impactado sus deseos de independencia y han tenido que postergarla. Desean y quieren ser independientes, pero debido a las condiciones económicas no lo pueden hacer; frente a ello, juegan con la ecuación de una independencia moral con la dependencia económica; situación que los tensiona. La universidad, como institución que los conecta, les da la independencia moral, pero no es suficiente para que ellos puedan resolver su propia independencia económica. 
La mirada al futuro

La idea del futuro y el logro de los sueños imaginados van generando trayectorias lineales; sin embargo, las oportunidades y elecciones que se tienen para realizarla pueden fragmentarla, observándose, por ejemplo, que cada vez más los jóvenes viven una adultez forzada, debido a que se $\operatorname{casan}^{12}$ o asumen el rol de jefes de hogar en ausencia de los padres. Dicha adultez forzada, cambia las condiciones de vida, los sueños y los sentidos del mundo que se imaginan para el futuro.

De los jóvenes que participaron en el grupo focal, por lo menos hay cuatro historias que se distinguen por ello, y que recogen un sentir de empatía por parte de los otros integrantes. Cuando se les preguntó cuáles eran aquellos sueños que se imaginaban para este momento de su vida que no los hubieran cumplido, un par de respuestas evidenciaban que ni siquiera tenían contemplado ir a la universidad; más bien fueron los consejos y las presiones de otros actores sociales que los animaron a estudiar, y ya ahora, se dan cuenta de lo importante que les ha sido; situación que es apropiada como indudable por los otros miembros. Importancia de contar con estudios universitarios, no solo por el conocimiento, sino porque les otorga una posición diferente en la estructura social que les permite acercarse de distinta manera al mundo social; estrechar las distancias sociales. Tienen conciencia de que dicho prestigio tiene que ver con el hecho de que ellos se visualicen como conectados; privilegio, estatus y poder simbólico que les otorga el haber estudiado:

Cuando era pequeña me negaba a ser maestra, me gustaba ser maestra de niño de primaria o de kínder, sin embargo... por cuestiones del destino cambiaron y creo que para bien, ... el estudiar la licenciatura en políticas me ha abierto un panorama mucho más grande (Sonia).

Bueno yo no imaginé que iba a estudiar en la universidad, porque, desde los 11 años dejé de vivir con mis papás (Ana).

Sin embargo, dichos sueños imaginados y en su caso realizados, tienen que ver con las coyunturas que les imposibilitan o les ayudan a cumplirlos. Dichas coyunturas no son casuales, aunque ellos las asuman de dicha manera. Los sueños se cumplen por sus capitales, sus procesos de empodera-

12 En el estudio de Oliveira (2008) se muestra que en el estrato bajo las mujeres jóvenes de 15 a 19 años ya entraron a trabajar en mayor medida que en las del estrato medio-alto, estas últimas tienen mayor presencia en que las primeras en el grupo de 25 a 29. Esto último responde a que en los estratos bajos en mayor proporción y edades más tempranas las jóvenes ya han experimentado la unión de pareja y han iniciado su ciclo reproductivo. 
miento, los contextos y actores que les ayudan y por las oportunidades y accesos que tienen en la medida que sufren de menor violencia estructural.

En ese sentido, los egresados aprueban que cada vez más sus sueños se van postergando y aplazando a razón de darle respuesta a sus necesidades básicas más apremiantes como la alimentación; dejan para un futuro mediano aquellos sueños como el seguir estudiando.Y ello como posibilidad de una vida más cómoda, no sólo porque pueden recibir algunas becas, sino porque en la escuela encuentran el mayor espacio para socializar y manifestar su identidad.

En realidad, en los momentos que vas viviendo siempre hay oportunidades y decisiones que vas a tomar y también hay otros sueños que no cumplí... encontrar un trabajo en el cual me permitan hacer lo que yo sé hacer... que me paguen bien, porque eso me permitiría seguir haciendo más proyectos, más ideas y que día a día voy generando y sí he dejado como sueños a posteriori (Juan).

Ejemplo de ello son las respuestas que pronuncian cuando se habla del tiempo libre; este desaparece con el vaivén de los mercados laborales. Pocos hacen mención de pasar un buen tiempo con los amigos; más bien hacen referencia a conectarse con otros mecanismos tecnológicos: escuchar música, consultar las redes, etcétera.

En cuanto a actividades de tiempo, ya dejando de lado lo laboral, me gusta leer, dormir también, me gusta mucho dormir, aprovechar ese espacio de descanso que si no después siento que no rindo para nada... trato de buscar ese espacio para estar sola y comenzar no sé, investigar en internet... (Judith).

Los sueños dibujan no solo la temporalidad del sujeto, sino que en ellos se reconocen los procesos de inflexión que cambian las trayectorias, que enseñan las crisis y las estrategias para afrontarlas. Un común denominador, en los egresados estudiados, es el sueño a largo plazo de fundar, instrumentar y trabajar en organizaciones de la sociedad civil para elevar la calidad de vida de la población en general.Actividad que aparece en el pasado para replantearse en el futuro. Representación que nos deja sospechar que tienen conciencia de la desigualdad que están viviendo a consecuencia de las crisis y la violencia estructural, que se saben que pueden actuar como agentes sociales para el cambio, pero que la propia violencia que viven los deja inhabilitados para asumirse como dichos agentes:

Ya de manera general en la conclusión de sueños, crear mis propias empresas... En mi, caso estando establecido, hacer programas sociales que ayuden a la gente a la educación 
civiles, artísticos, esa cuestión me gustaría mucho desempeñarla si se diera la oportunidad (Dionisio).

Lo cierto es que los jóvenes registran dificultades al momento de plantear estrategias que les hagan romper con la violencia estructural, aunque se sienten privilegiados por contar con capitales cognitivos que les dan herramientas distintas frente a otro tipo de jóvenes. Si bien les han hecho creer que a través de actos volutivos pueden romper con dicha desigualdad, tienen conciencia de que solo a partir de transformar situaciones estructurales se podrá mejorar su calidad de vida.

\section{CONCLUSIONES}

La violencia estructural, de la que son objeto algunos jóvenes, se materializa en los propios proyectos de vida y la construcción de los sentidos sociales que se hace de su tránsito a los mercados profesionales y a las oportunidades que se visualizan en ellos. Sin embargo, a pesar de haber transitado por la universidad, las desigualdades, están reconfigurando los sentidos y prácticas culturales que realizan para hacerle frente a estas.

Los jóvenes egresados quieren ser independientes de su núcleo familiar; sin embargo, económicamente no pueden. Dicho conflicto entre sentirse moralmente independientes y no así económicamente, los obliga a construir estrategias de convivencia ya sea con la familia o con amigos. Estrategias que les ayudan a salir adelante frente a la precariedad económica, aunque, por ejemplo, el hecho de vivir en núcleos familiares extensos les provoca un dejo de insatisfacción.

El empleo y el trabajo profesional se ven como un mecanismo para lograr dicha independencia; sin embargo, no encuentran trabajos lo suficientemente acordes con su formación profesional, mucho menos bien remunerados. Por ello mismo, reclaman mayores empleos de calidad en aras de contar con mayores oportunidades de realización profesional y con las condiciones para vivir con calidad.

También es interesante observar que los estudios de grado se observan como metas a medio plazo. Por una parte, porque la universidad ha sido el espacio por excelencia para socializar y reproducir sus prácticas, que les dan identidad; por otra parte, porque le adjudican a la universidad y al acto de seguir estudiando, la propiedad de ser el eje de oportunidades, alternativas y accesos a mejores condiciones de vida. 
La universidad se convierte en el espacio por excelencia en donde ellos han socializado y construido, a partir de las prácticas, su identidad; egresar de dicho espacio, les implica dejar de seguir reproduciendo las prácticas y asumir otras que les conducen al mundo adulto; un mundo donde visualizan más la precariedad que la comodidad. Pensar en regresar a las aulas, es también, recuperar sus relaciones sociales y lógicas de reconocimiento que los hacen sentir integrados. Si bien, visibilizan en los estudios de grado mayores oportunidades para la movilidad social y profesional, también ven en ellos, la recuperación de su capital social y simbólico para relacionarse con las instituciones y con sus pares. Relaciones que les dan identidad e integración, pero que al mismo tiempo les colocan en las redes de las alternativas y accesos para reinventarse una vida mejor.

Repasan la idea de confrontar la violencia estructural de la que son objeto (autoevaluada por ellos mismos como falta de oportunidades laborales y profesionales, repercutiendo en su independencia y espacios de recreación), a partir de insertarse en otro tipo de lógicas que les den mayores recursos para moverse socialmente, en tanto la licenciatura les ha sido insuficiente para enfrentar las desigualdades que experimentan en su cotidiano. Una de ellas es imaginar a largo plazo el trabajo con grupos y organizaciones de la sociedad civil.

Los proyectos de vida, los sueños, se van postergando porque las condiciones estructurales no les están permitiendo contar con opciones para elegir; más bien se someten a los vaivenes del mercado laboral (y no profesional) al grado de notarse un tipo de desencanto por el futuro. Los sueños, construyen los sentidos sociales; en ellos se visualizan lo que esperan los jóvenes de un futuro inmediato y a mediano plazo; los sueños a largo plazo se ven en la esfera de lo utópico, imaginando a muy largo plazo su ejercicio y capacidad como agentes de cambio.

Los resultados que aquí se presentan dejan ver, por una parte, que los jóvenes hacen un reclamo a mejores condiciones de vida, no solo para ellos, sino para la población en general; por otra, reconocen la violencia estructural de la que son objeto, aunque les es difícil asumirla. Por ello mismo, aunque estén de becarios, con trabajos sin prestaciones o en casa, construyen estrategias enunciativas y de auto-representación, justificando que están haciendo algo; actividad que se ciñe al constructo de la suerte en función de otros. Suerte que les permite justificar su quehacer, a partir de la actividad que realizan, como el escenario para alcanzar y cumplir sus sueños. Sueños que son truncados o imaginados a mediano plazo. Olvidan que no es cuestión de suerte, sino de oportunidades y recursos en la gene- 
ración de calidad de vida y en la disminución de la desigualdad social. Olvidan que la educación por sí sola no cambia las condiciones de vida, sino que es el entramado de las estructuras sociales las que permean mayores o menores desigualdades sociales.

Habría que poner especial atención en el diseño de políticas públicas a propósito de los jóvenes que viven la desigualdad y que se encuentran en arenas movedizas entre los mercados profesionales y laborales precarios y la calidad de vida; arenas a partir de las cuales se crean y recrean como actores sociales de cambio.

\section{BIBLIOGRAFÍA}

Alonso, L. E. (1998) «La mirada cualitativa en sociología», Madrid, Fundamentos.

Cisneros, J. L. (2007): «Cultura, juventud y delincuencia en el Estado de México», Papeles de Población, 52, pp. 255-280

Duarte, Q. K. (2000): « ¿Juventud o juventudes? Acerca de cómo mirar y remirar a las juventudes de nuestro continente», Última Década, 13, pp. 59-77.

GALTUNG, J. (1995): Investigaciones teóricas: sociedad y cultura contemporáneas, Madrid, Tecnos.

Makowski, S. (2010): Jóvenes que viven en la calle, México, Universidad Autónoma Metropolitana, Iztapalapa-Siglo XXI.

Oliveira, O. de y Minor Mora, S. (2008): «Desigualdades sociales y transición a la adultez en el México contemporáneo», Papeles de Población, 57, pp. 117-152.

RAmírez, J. C. (2010): «Violencia y jóvenes. Enclaves de la masculinidad» en Reguillo, R. (coord.) (2010): Los jóvenes en México, México, Fondo de Cultura Económica-Consejo Nacional para la Cultura y las Artes.

Reguillo, R. (coord.) (2010): Los jóvenes en México, México, Fondo de Cultura Económica-Consejo Nacional para la Cultura y las Artes.

Reguillo, R. (1997): «Jóvenes: la construcción del enemigo», Revista Latinoamericana de Comunicación CHASQUI, 60.

Reguillo, R. (2007): «Legitimidad (es) divergentes» en Jóvenes Mexicanos. Encuesta Nacional de la Juventud 2005, México, SEP, pp. 77-133.

SuÁrez, M. H. (2010): «Desafíos de una relación en crisis. Educación y jóvenes mexicanos» en Reguillo, R. (coord.) (2010): Los jóvenes en México, 
México, Fondo de Cultura Económica-Consejo Nacional para la Cultura y las Artes.

Tortosa, J. M. (2012), Desigualdad, conflicto, violencia. Cinco ensayos sobre la realidad mundial, Ecuador, PYDLos ediciones - Universidad de Cuenca.

UAEM (2004), Licenciatura en Comunicación. Currículum 2004, Facultad de Ciencias Políticas y Administración Pública:Toluca.

UAEM (2004), Licenciatura en Sociología. Currículum 2004, Facultad de Ciencias Políticas y Administración Pública:Toluca.

Urteaga, M. (2010): «Género, clase y etnia. Los modos de ser joven» en Reguillo, R. (coord.) (2010): Los jóvenes en México, México, Fondo de Cultura Económica-Consejo Nacional para la Cultura y las Artes.

\section{DOCUMENTOS Y RECURSOS ELECTRÓNICOS}

CONAPO (2010), Índice de marginación por localidad 2010. Disponible en http://www.conapo.gob.mx/es/CONAPO/Indice_de_Marginacion_por_ Localidad_2010 [Consultado el 22 de noviembre de 2014].

IMJUVE (2010), Encuesta Nacional de Juventud 2010. Resultados generales.

Disponible en: http://www.imjuventud.gob.mx/imgs/uploads/Encuesta_Nacional_de_Juventud_2010_-_Resultados_Generales_18nov11.pdf [22 de noviembre de 2014].

- (2012), Encuesta Nacional de Valores en Juventud 2012. Disponible en: Imjuve. http://www.imjuventud.gob.mx/imgs/uploads/ENVAJ_2012.pdf [Consultada el 22 de abril de 2014].

INEGI (2012), Estadísticas a propósito del día internacional de la juventud.

Disponible en http://www.inegi.org.mx/inegi/contenidos/espanol/ prensa/contenidos/estadisticas/2012/juventud12.asp? $\mathrm{c}=2844 \& \mathrm{ep}=99$ [Consultada el 18 de abril de 2014].

- (2012), Encuesta de la Comunidad Americana 2010, Disponible en http://www.census.gov/newsroom/releases/archives/american_community_survey_acs/cb11-158sp.html [Consultada el 23 de abril de 2014].

La Jornada «Sin lugar en la UnAm, 87\% de los aspirantes», 13 de julio de 2013. Disponibleenhttp://www.jornada.unam.mx/2013/07/15 politica/002n1pol [Consultada el 12 de mayo de 2014].

La Jornada, «El desempleo, mayor en jóvenes de clases medias o altas y con más estudios: experto», 21 de mayo de 2014. Disponible en http://www. 
jornada.unam.mx/2014/05/21/politica/014n1pol [Consultada el 30 de mayo de 2014].

La Jornada, «Los mejor preparados académicamente, los más afectados», 20 de mayo de 2014. Disponible en http://www.jornada.unam. $\mathrm{mx} / 2014 / 05 / 20 /$ sociedad/035n2soc [Consultada el 20 de mayo de $2014]$.

La Jornada, «Rechazado, 92\% de aspirantes a las licenciaturas en la UNAM», 11 deabrilde 2013.Disponibleenhttp://www.jornada.unam.mx/2013/04/11/ politica/007n1pol [Consultada el 12 de mayo de 2014].

oCDE (2013), Panorama Educativo. Disponible en http://www.oecd-ilibrary. org/education/panorama-de-la-educacion-2013-indicadores-de-la-ocde eag-2013-es [Consultado el 22 de mayo de 2014].

Oliveira, O. de (2014), «Jóvenes en una sociedad desigual», conferencia en El Colegio de México el día 1 de mayo en http://www.colef. $\mathrm{mx} /$ ?evento=jovenes-en-una-sociedad-desigual-trayectorias-de-vidacontrastantes\&lang=es [Consultada el 3 de junio de 2014]. 